\title{
GREEDY STRATEGIES FOR CONVEX OPTIMIZATION
}

\author{
HAO NGUYEN AND GUERGANA PETROVA
}

\begin{abstract}
We investigate two greedy strategies for finding an approximation to the minimum of a convex function $E$ defined on a Hilbert space $H$. We prove convergence rates for these algorithms under suitable conditions on the objective function $E$. These conditions involve the behavior of the modulus of smoothness and the modulus of uniform convexity of $E$.

Key Words: Greedy Algorithms, Convex Optimization, Rates of Convergence.
\end{abstract}

\section{INTRODUCTION}

Convex optimization has many application domains such as automatic control systems, signal processing, communications and networks, electronic circuit design, data analysis and modeling, statistical estimation, finance, and combinatorial optimization. A general description for convex optimization is that we are given a Banach space $X$ and a convex function $E$ on $X$ whose minimum we wish to compute. Thus, we are interested in the development and analysis of algorithms for approximating

$$
\inf _{x \in D} E(x),
$$

where $D$ is a convex subset of $X . E$ is called the objective function and, by the convexity assumption, satisfies the condition

$$
E(\gamma x+\delta y) \leq \gamma E(x)+\delta E(y), \quad x, y \in D, \quad \gamma, \delta \geq 0, \quad \gamma+\delta=1 .
$$

The classical results on convex optimization deal with objective functions $E$ defined on subsets in $\mathbb{R}^{d}$ with moderate values of $d$, see e.g. [2]. However, several of the applications, listed above, lead to optimization on Banach spaces of dimension $d$, where $d$ is quite large or even $\infty$. The design of algorithms for such high dimensional problems is quite challenging, typical convergent results involve the dimension $d$ and suffer from the curse of dimensionality.

Recently, several researchers (see e.g. [7, 8, 11]), have proposed strategies for solving (1.1), where the curse of dimensionality is overcome by using greedy techniques, similar to those originally developed for the approximation of a given element $x \in X$.

This research was supported by the Office of Naval Research Contract ONR N00014-11-1-0712, by the NSF Grant DMS 1222715, and by the Bulgarian Science Fund Grant DFNI-T01/0001. 
The minimum in (1.1) is approximated by $E\left(x_{m}\right), m=0,1, \ldots$, where each $x_{m}$ is constructed as a linear combination of $m$ elements (i.e. $x_{m}$ is $m$ sparse) from a given dictionary $\mathcal{D}$. Recall that $\mathcal{D}$ is called a symmetric dictionary if each $\varphi \in \mathcal{D}$ has norm $\|\varphi\| \leq 1$, if $\varphi \in \mathcal{D}$, then $-\varphi \in \mathcal{D}$, and the closure of $\operatorname{span} \mathcal{D}$ is $X$. A typical a priori convergence result given by the above authors for these greedy algorithms is proven under two assumptions:

(i) An assumption on the smoothness of $E$.

(ii) An assumption that the minimum in (1.1) is taken at a point $\bar{x}$ which is in the convex hull of the dictionary $\mathcal{D}$.

In this paper, we investigate the special case when $X=H$ is a Hilbert space, the dictionary $\mathcal{D}=\left\{ \pm \varphi_{j}\right\}_{j=1}^{\infty}$, where $\left\{ \pm \varphi_{j}\right\}_{j=1}^{\infty}$ is an orthonormal basis, and $D=H$ (which corresponds to global minimization). We assume that the global minimum is attained at some point $\bar{x} \in H$. It follows then that the minimum is taken on the set

$$
\Omega:=\{x \in H: E(x) \leq E(0)\} .
$$

We assume throughout this paper that the set $\Omega$ is bounded in $H$. We impose the following assumptions on the objective function $E$ :

Condition 0: $E$ has a Frechet derivative $E^{\prime}(x) \in H$ at each point $x$ in $\Omega$ and

$$
\left\|E^{\prime}(x)\right\| \leq M_{0}, \quad x \in \Omega
$$

where throughout $\|\cdot\|$ denotes the norm on $H$.

Condition 1: There are constants $0<\alpha, 1<q \leq 2$ and $0<M$, such that for all $x, x^{\prime}$ with $\left\|x-x^{\prime}\right\| \leq M, x \in \Omega$,

$$
E\left(x^{\prime}\right)-E(x)-\left\langle E^{\prime}(x), x^{\prime}-x\right\rangle \leq \alpha\left\|x^{\prime}-x\right\|^{q} .
$$

Condition 2: There are constants $0<\beta, 2 \leq p<\infty$ and $0<M$, such that for all $x, x^{\prime}$ with $\left\|x-x^{\prime}\right\| \leq M, x \in \Omega$,

$$
E\left(x^{\prime}\right)-E(x)-\left\langle E^{\prime}(x), x^{\prime}-x\right\rangle \geq \beta\left\|x^{\prime}-x\right\|^{p} .
$$

We show in $\$ 2$ that Condition 1 is equivalent to conditions on the modulus of smoothness $\rho(E, u)$, and Condition 2 is equivalent to conditions on the modulus of uniform convexity $\delta_{1}(E, u)$, as usually defined in convex optimization (see e.g. [10]), and introduced by us in $\$ 2$.

We study two greedy procedures for solving (1.1). The first is the analogue for convex minimization of the Orthogonal Matching Pursuit Algorithm used for approximation (see [6]). We denote this convex minimization algorithm by $\mathrm{OMP}(\mathrm{co}) 1$.

\footnotetext{
${ }^{1}$ Here and later we will use the abbreviation (co) if an algorithm is used for convex optimization
} 
The second is the Weak Chebyshev Greedy Algorithm (WCGA(co)) as introduced by Temlyakov [8]. These greedy procedures, which are defined in $\$ 3$, iteratively generate a sequence $x_{m}, m=0,1, \ldots$, where each $x_{m}$ is $m$ sparse, and then use $E\left(x_{m}\right)$ as the approximation to the minimum $E(\bar{x})$.

Our main results are Theorem 4.5 and Theorem 4.6 which establish a priori convergence rates for both $\operatorname{OMP}(\mathrm{co})$ and the $\operatorname{WCGA}(\mathrm{co})$ when they are used to find the minimum of a function $E$ that satisfies Conditions $\mathbf{0}, \mathbf{1}$ and $\mathbf{2}$. For example, we show that if the objective function $E$ satisfies Condition $\mathbf{0}$ and Condition 1, is strongly convex on $H$ (therefore satisfies Condition 2 with $p=2$ ), and its minimizer $\bar{x}$ is sparse with respect to $\mathcal{D}$, then the error at the $m$-th step of the $\mathrm{OMP}(\mathrm{co})$ satisfies the inequality

$$
E\left(x_{m}\right)-E(\bar{x}) \leq C_{0} m^{1-\frac{q}{2-q}}, \quad 1<q<2,
$$

and

$$
\left\|x_{m}-\bar{x}\right\| \leq C_{1} m^{\frac{1}{2}-\frac{q}{2(2-q)}}
$$

where $C_{0}=C_{0}(q, E)$ and $C_{1}=C_{1}(q, E)$. We also prove exponential convergence in the case $q=2$. In contrast, the results from [8] and [11] do not impose Condition 2 and only give the rate $1-q$. In summary, we show that imposing more conditions on the convexity of the objective function $E$ (like Condition 2) results in provably improved convergence rates for both $\mathrm{OMP}(\mathrm{co})$ and WCGA(co).

\section{Conditions on E}

In this section, we discuss the compatibility of the conditions (Condition $\mathbf{0}$, Condition 1 and Condition 2) imposed on the objective function $E$ and their relation to the modulus of smoothness and modulus of uniform convexity of $E$. We recall that a function $E$ is Frechet differentiable at $x \in \Omega$ if there exists a bounded linear functional, denoted by $E^{\prime}(x)$, such that

$$
\lim _{h \rightarrow 0} \frac{\left|E(x+h)-E(x)-\left\langle E^{\prime}(x), h\right\rangle\right|}{\|h\|}=0 .
$$

We start with discussing the connection between Condition $\mathbf{1}$ and the modulus of uniform smoothness of $E$ on $\Omega$.

\subsection{Condition 1.}

Given a convex function $E: H \rightarrow \mathbb{R}$ and a set $S \subset H$, the modulus of smoothness of $E$ on $S$ is defined by

$$
\rho(E, u):=\rho(E, u, S):=\frac{1}{2} \sup _{x \in S,\|y\|=1}\{E(x+u y)+E(x-u y)-2 E(x)\}, \quad u>0,
$$


and the modulus of uniform smoothness of $E$ on $S$ is defined by

$$
\rho_{1}(E, u, S):=\sup _{x \in S,\|y\|=1, \lambda \in(0,1)}\left\{\frac{(1-\lambda) E(x-\lambda u y)+\lambda E(x+(1-\lambda) u y)-E(x)}{\lambda(1-\lambda)}\right\} .
$$

These two moduli of smoothness are equivalent (see [10], page 205):

Lemma 2.1. Let $E$ be a convex function defined on $H$, and let $S \subset H$, then

$$
4 \rho\left(E, \frac{u}{2}, S\right) \leq \rho_{1}(E, u, S) \leq 2 \rho(E, u, S) .
$$

The next lemma shows the relation between the modulus of uniform smoothness and Condition 1.

Lemma 2.2. Let $E$ be a convex function defined on a Hilbert space $H$ and $E$ be Frechet differentiable on a set $S \subset H$. The following statements are equivalent for any $q \in(1,2]$ and $M>0$.

(i) There exists $\alpha>0$, such that for all $x \in S, x^{\prime} \in H,\left\|x-x^{\prime}\right\| \leq M$,

$$
E\left(x^{\prime}\right)-E(x)-\left\langle E^{\prime}(x), x^{\prime}-x\right\rangle \leq \alpha\left\|x^{\prime}-x\right\|^{q} .
$$

(ii) There exists $\alpha_{1}>0$, such that

$$
\rho(E, u, S) \leq \alpha_{1} u^{q}, \quad 0<u \leq M .
$$

The same result holds with $\rho$ replaced by $\rho_{1}$.

Proof. While this is a particular case of Corollary 3.5.7 from [10], for completeness of this paper, we provide a simple proof of this lemma. First, observe that because of Lemma 2.1, statement (ii) for $\rho$ and $\rho_{1}$ are equivalent, and so we can use them interchangeably. Assume that the first statement is true. For any $x \in S, y \in H$, $\|y\|=1$ and any $0<u \leq M$, let $x^{\prime}:=x+u y, x^{\prime \prime}:=x-u y$. Then, we have $\left\|x-x^{\prime}\right\|=u \leq M,\left\|x^{\prime \prime}-x\right\|=u \leq M$. We apply (2.77) for the pairs $\left(x^{\prime}, x\right)$ and $\left(x^{\prime \prime}, x\right)$ to obtain

$$
E(x+u y)-E(x)-u\left\langle E^{\prime}(x), y\right\rangle \leq \alpha u^{q}, \quad E(x-u y)-E(x)+u\left\langle E^{\prime}(x), y\right\rangle \leq \alpha u^{q} .
$$

Therefore, we have

$$
E(x+u y)+E(x-u y)-2 E(x) \leq 2 \alpha u^{q} .
$$

We take the supremum over $x \in S, y \in H,\|y\|=1$ and derive $\rho(E, u, S) \leq \alpha u^{q}$, $0<u \leq M$, which gives the lemma for $\rho$.

Conversely, suppose that (ii) holds for $\rho_{1}$. Then, for any $\lambda \in(0,1)$ and any $x \in S, y \in H,\|y\|=1,0<u \leq M$,

$$
\frac{(1-\lambda) E(x-\lambda u y)+\lambda E(x+(1-\lambda) u y)-E(x)}{\lambda(1-\lambda)} \leq \alpha_{1} u^{q} .
$$


This is the same as saying

$$
\frac{E(x-\lambda u y)-E(x)}{(1-\lambda) \lambda}+\frac{E(x+(1-\lambda) u y)-E(x-\lambda u y)}{1-\lambda} \leq \alpha_{1} u^{q} .
$$

We let $\lambda \rightarrow 0^{+}$and use the continuity of $E$ and the definition of Frechet derivative $E^{\prime}(x)$ with $h=-\lambda u y$, to obtain

$$
\left\langle E^{\prime}(x),-u y\right\rangle+E(x+u y)-E(x) \leq \alpha_{1} u^{q} .
$$

Now, for any $x \in S, x^{\prime} \in H,\left\|x^{\prime}-x\right\| \leq M$, we let $u=\left\|x^{\prime}-x\right\|, y=\frac{x^{\prime}-x}{\left\|x^{\prime}-x\right\|}$. The above inequality can be written as

$$
E\left(x^{\prime}\right)-E(x)-\left\langle E^{\prime}(x), x^{\prime}-x\right\rangle \leq \alpha_{1}\left\|x^{\prime}-x\right\|^{q},
$$

which is (2.7) with $\alpha=\alpha_{1}$.

\subsection{Condition 2.}

We first observe the following:

Claim 1. If Condition 2 holds for a convex function $E$ and a set $\Omega$ that is convex and bounded, then Condition 2 holds for all $x, x^{\prime} \in \Omega$ with $\beta$ replaced by $\beta_{0}>0$.

Proof. Since $\Omega$ is bounded, there is $L>0$, such that $\operatorname{diam}(\Omega) \leq L M$. Let $x, x^{\prime} \in \Omega$. If $\left\|x-x^{\prime}\right\| \leq M$, Condition 2 holds for the pair $\left(x, x^{\prime}\right)$ provided $\beta_{0} \leq \beta$. If $\left\|x-x^{\prime}\right\|>M$, we chose a point $x_{1}$, such that

$$
x_{1}=\gamma x^{\prime}+(1-\gamma) x \in \Omega, \quad \gamma:=\frac{M}{\left\|x-x^{\prime}\right\|} \geq L^{-1} .
$$

Clearly $\left\|x-x_{1}\right\|=M$, and therefore

$$
E\left(x_{1}\right)-E(x)-\left\langle E^{\prime}(x), x_{1}-x\right\rangle \geq \beta\left\|x_{1}-x\right\|^{p} .
$$

Because of the convexity of $E$,

$$
E\left(x_{1}\right)-E(x) \leq \gamma\left[E\left(x^{\prime}\right)-E(x)\right]
$$

A combination of the last two inequalities and the fact that $x_{1}-x=\gamma\left(x^{\prime}-x\right)$ result in

$$
E\left(x^{\prime}\right)-E(x)-\left\langle E^{\prime}(x), x^{\prime}-x\right\rangle \geq \beta \gamma^{p-1}\left\|x^{\prime}-x\right\|^{p} \geq \beta L^{1-p}\left\|x^{\prime}-x\right\|^{p} .
$$

Therefore, the claim has been proven with $\beta_{0}=\min \left\{\beta, \beta L^{1-p}\right\}$.

Note that Condition $\mathbf{2}$ is a generalization of the notion of strongly convex functions. Recall that a function $E$ is called strongly convex on $H$, if there is a constant $\beta>0$, called the convexity parameter of $E$, such that

$$
E\left(x^{\prime}\right)-E(x)-\left\langle E^{\prime}(x), x^{\prime}-x\right\rangle \geq \beta\left\|x^{\prime}-x\right\|^{2}, \quad x, x^{\prime} \in H .
$$

Next, we discuss the compatibility between the convexity of $E$ and Condition 2. 
Lemma 2.3. Let $E$ be a Frechet differentiable function on $H$. $E$ is convex on $H$ if and only if

$$
E\left(x^{\prime}\right)-E(x)-\left\langle E^{\prime}(x), x^{\prime}-x\right\rangle \geq 0, \quad \text { for all } x, x^{\prime} \in H, \quad\left\|x-x^{\prime}\right\| \leq M .
$$

Proof. For convex functions on $\mathbb{R}^{n}$, a proof (without the restriction $\left\|x^{\prime}-x\right\| \leq M$ ) can be found in [2]. Simple modifications of this proof (which we do not give) result in a proof of the lemma.

Finally, we present a concept which is dual to the modulus of uniform smoothness for convex functions, called the modulus of uniform convexity (see [1, 10]) and show how it is related to Condition 2. Given a convex function $E: H \rightarrow \mathbb{R}$ and a set $S \subset H$, its modulus of uniform convexity on $S$ is defined by

$$
\delta_{1}(E, u, S):=\inf _{x \in S,\|y\|=1, \lambda \in(0,1)}\left\{\frac{(1-\lambda) E(x-\lambda u y)+\lambda E(x+(1-\lambda) u y)-E(x)}{\lambda(1-\lambda)}\right\} .
$$

We prove a lemma (see [10]) that shows the equivalence of Condition 2 and certain behavior of the modulus of uniform convexity $\delta_{1}$ of $E$.

Lemma 2.4. Let $E$ be a convex function defined on a Hilbert space $H$ and $E$ be Frechet differentiable on $S \subset H$. The following statements are equivalent for any $p \in[2, \infty)$ and $M>0$.

(i) There exists $\beta>0$, such that for all $x \in S, x^{\prime} \in H,\left\|x-x^{\prime}\right\| \leq M$,

$$
E\left(x^{\prime}\right)-E(x)-\left\langle E^{\prime}(x), x^{\prime}-x\right\rangle \geq \beta\left\|x^{\prime}-x\right\|^{p} .
$$

(ii) There exists $\beta_{1}>0$, such that

$$
\delta_{1}(E, u, S) \geq \beta_{1} u^{p}, \quad 0<u \leq M .
$$

Proof. Assume that the first statement is true. For any $x \in S, y \in H,\|y\|=1$, $0<u \leq M$ and $\lambda \in(0,1)$, let $x^{\prime}:=x-\lambda u y, x^{\prime \prime}:=x+(1-\lambda) u y$. Then, we have $\left\|x-x^{\prime}\right\|=\lambda u \leq M,\left\|x^{\prime \prime}-x\right\|=(1-\lambda) u \leq M$. We apply (2.10) for $x \in S, x^{\prime} \in H$ and $x \in S, x^{\prime \prime} \in H$ to derive

$$
\begin{gathered}
E(x-\lambda u y)-E(x)+\lambda u\left\langle E^{\prime}(x), y\right\rangle \geq \beta \lambda^{p} u^{p}, \\
E(x+(1-\lambda) u y)-E(x)-(1-\lambda) u\left\langle E^{\prime}(x), y\right\rangle \geq \beta(1-\lambda)^{p} u^{p} .
\end{gathered}
$$

Multiplying the first inequality by $(1-\lambda)$, the second one by $\lambda$ and adding them yields

$$
(1-\lambda) E(x-\lambda u y)+\lambda E(x+(1-\lambda) u y)-E(x) \geq \beta \lambda(1-\lambda)\left(\lambda^{p-1}+(1-\lambda)^{p-1}\right) u^{p} .
$$

Since $\lambda^{p-1}+(1-\lambda)^{p-1} \geq 2^{2-p}$ for $\lambda \in(0,1)$, we have

$$
\frac{(1-\lambda) E(x-\lambda u y)+\lambda E(x+(1-\lambda) u y)-E(x)}{\lambda(1-\lambda)} \geq 2^{2-p} \beta u^{p} .
$$


We take the infimum over $x \in S, y \in H,\|y\|=1$ and $\lambda \in(0,1)$ and obtain that $\delta_{1}(E, u, S) \geq 2^{2-p} \beta u^{p}, 0<u \leq M$, which is (2.11) with $\beta_{1}=2^{2-p} \beta$.

Conversely, suppose that for some $\beta>0$ we have $\delta_{1}(E, u, S) \geq \beta u^{p}$ for all $0<u \leq$ $M$. It follows from the definition of $\delta_{1}$ that for any $\lambda \in(0,1), x \in S, y \in H,\|y\|=1$ and $0<u \leq M$,

$$
\frac{(1-\lambda) E(x-\lambda u y)+\lambda E(x+(1-\lambda) u y)-E(x)}{\lambda(1-\lambda)} \geq \beta_{1} u^{p}
$$

This is the same as saying

$$
\frac{E(x-\lambda u y)-E(x)}{\lambda}+\frac{E(x+(1-\lambda) u y)-E(x)}{1-\lambda} \geq \beta_{1} u^{p} .
$$

We let $\lambda \rightarrow 0^{+}$and by the continuity of $E$ and the definition of Frechet derivative $E^{\prime}(x)$ for $h=-\lambda u y$, we obtain

$$
\left\langle E^{\prime}(x),-u y\right\rangle+E(x+u y)-E(x) \geq \beta_{1} u^{p} .
$$

Now, for any $x \in S, x^{\prime} \in H,\left\|x^{\prime}-x\right\| \leq M$, we let $u=\left\|x^{\prime}-x\right\|, y=\frac{x^{\prime}-x}{\left\|x^{\prime}-x\right\|}$ and derive

$$
E\left(x^{\prime}\right)-E(x)-\left\langle E^{\prime}(x), x^{\prime}-x\right\rangle \geq \beta_{1}\left\|x^{\prime}-x\right\|^{p},
$$

which is (2.10) with $\beta=\beta_{1}$.

2.3. The conditions on $E$ and their connection to Compressed Sensing. Let us summarize that as a result of Lemma 2.2 and Lemma 2.4, we have proven the following.

Lemma 2.5. Let $E$ be a convex function defined on a Hilbert space $H$. Let us denote by $\Omega$ the set $\Omega=\{x \in H: E(x) \leq E(0)\}$ and $E$ be Frechet differentiable on $\Omega$. Let $\delta_{1}(E, \cdot, \Omega)$ and $\rho_{1}(E, \cdot, \Omega)$ be the modulus of uniform convexity and modulus of uniform smoothness of $E$ on $\Omega$, respectively. The following two statements are equivalent

(i) E satisfies Condition 1 and Condition 2.

(ii) There exist constants $\alpha_{1}>0, \beta_{1}>0$, such that

$$
\beta_{1} u^{p} \leq \delta_{1}(E, u, \Omega) \leq \rho_{1}(E, u, \Omega) \leq \alpha_{1} u^{q}, \quad u \in(0, M] .
$$

Let us next observe that (i) of the above lemma has a similar flavor to conditions that are imposed in compressed sensing. Indeed, conditions similar to Condition 1 and Condition 2 have been considered by Zhang in [12, where he solves a sparse optimization problem in $\mathbb{R}^{n}$, using greedy based strategies. He considers any convex function $E$ on $\mathbb{R}^{n}$ for which there are constants $\alpha(s), \beta(s)>0$ such that

$$
\beta(s)\left\|x^{\prime}-x\right\|_{2}^{2} \leq E\left(x^{\prime}\right)-E(x)-\left\langle E^{\prime}(x), x^{\prime}-x\right\rangle \leq \alpha(s)\left\|x^{\prime}-x\right\|_{2}^{2},
$$


holds whenever $x, x^{\prime} \in \mathbb{R}^{n}$ and $x-x^{\prime}$ has $\leq s$ nonzero coordinates. Notice, that (2.12) is the same as our Condition 1 and Condition $\mathbf{2}$ except that it is only required to hold whenever $x-x^{\prime}$ is $s$ sparse whereas in our case we require this to hold for all $x, x^{\prime}$ with $\left\|x-x^{\prime}\right\| \leq M$. Zhang applied his results to the decoding problem in compressed sensing in which case $E(x)=\|A x-b\|_{2}^{2}$, and $A$ is a given $k \times n$ matrix with $k<<n$. For this choice of $E$, the Frechet derivative $E^{\prime}(x)$ can be computed explicitly as $\left\langle E^{\prime}(x),.\right\rangle=2\left\langle A^{T}(A x-b),.\right\rangle$. Moreover, we have

$$
\begin{aligned}
E\left(x^{\prime}\right)-E(x)-\left\langle E^{\prime}(x), x^{\prime}-x\right\rangle & =\left\|A x^{\prime}-b\right\|_{2}^{2}-\|A x-b\|_{2}^{2}-2\left\langle A^{T}(A x-b), x^{\prime}-x\right\rangle \\
& =\left\|A x^{\prime}-A x\right\|_{2}^{2}=\left\|A\left(x-x^{\prime}\right)\right\|_{2}^{2} .
\end{aligned}
$$

If we denote by $z=x^{\prime}-x$, condition (2.12) becomes

$$
\beta(s)\|z\|_{2}^{2} \leq\|A z\|_{2}^{2} \leq \alpha(s)\|z\|_{2}^{2},
$$

for $s$ sparse vectors $z \in \mathbb{R}^{n}$. This condition is known as the Restricted Isometry Property and was first introduced by Candes and Tao (see [3], [5]). For applications in compressed sensing one needs that $\alpha(s), \beta(s)$ are sufficiently close to one.

\section{GReEdy ALGORITHMS FOR optimization}

In this section, we introduce the two algorithms for convex minimization in a Hilbert space $H$ that we will analyze. As usual, we assume that $\left\{\varphi_{j}\right\}_{j=1}^{\infty}$ is an orthonormal basis for $H$. We begin with the $\mathrm{OMP}(\mathrm{co})$ algorithm.

\section{Orthogonal Matching Pursuit (OMP(co)):}

- Step 0: Define $x_{0}:=0$. If $E^{\prime}\left(x_{0}\right)=0$, stop the algorithm and define $x_{k}:=x_{0}$, $k \geq 1$.

- Step $m$ : Assuming $x_{m-1}$ has been defined and $E^{\prime}\left(x_{m-1}\right) \neq 0$, Find

$$
\varphi_{j_{m}}:=\operatorname{argmax}\left\{\left|\left\langle E^{\prime}\left(x_{m-1}\right), \varphi\right\rangle\right|, \varphi \in \mathcal{D}\right\},
$$

and define

$$
x_{m}:=\operatorname{argmin}_{x \in \operatorname{span}\left\{\varphi_{j_{1}}, \varphi_{j_{2}}, \ldots, \varphi_{j_{m}}\right\}} E(x) .
$$

If $E^{\prime}\left(x_{m}\right)=0$, stop the algorithm and define $x_{k}:=x_{m}, k>m$. Otherwise, go to Step $m+1$.

Note that if the algorithm stops at step $m$, then the output $x_{m}$ of the algorithm is the minimizer $\bar{x}$, because of the following well-known lemma.

Lemma 3.1. Let $E$ be a Frechet differentiable convex function, defined on a convex domain $\Omega$. Then $E$ has a global minimum at $\bar{x} \in \Omega$ if and only if $E^{\prime}(\bar{x})=0$. 
Weak Chebyshev Greedy Algorithm (WCGA(co)): The description of the WCGA(co) is the same as the $\operatorname{OMP}(\mathrm{co})$, with the only difference that a sequence $\left\{t_{k}\right\}_{k=1}^{\infty}, t_{k} \in(0,1]$ is used to weaken the condition on the choice of $\varphi_{j_{m}}$. Namely, $\varphi_{j_{m}}$ is now chosen to satisfy the inequality

$$
\left|\left\langle E^{\prime}\left(x_{m-1}\right), \varphi_{j_{m}}\right\rangle\right| \geq t_{m} \sup _{\varphi \in \mathcal{D}}\left\langle E^{\prime}\left(x_{m-1}\right), \varphi\right\rangle .
$$

When all $t_{k}=1, k \geq 1$, the WCGA(co) becomes the $\operatorname{OMP}(\mathrm{co})$.

Let us remark that neither of these two algorithms generates a unique sequence $x_{m}, m \geq 0$. The analysis that follows applies to any sequence generated by the corresponding algorithm.

For comparison with the results we prove in this paper, we recall the result of Temlyakov. Let $A_{1}(\mathcal{D})$ denote the closure (in $H$ ) of the convex hull of $\mathcal{D}$. The following theorem was proved in [8] in a more general setting of Banach spaces and general symmetric dictionaries.

Theorem 3.2 (8] Theorem 2.2). Let $E$ be a uniformly smooth convex function defined on a Banach space $X$ and let the set $\Omega:=\{x: E(x) \leq E(0)\}$ be bounded. Let the modulus of smoothness of $E$ on $\Omega$ satisfy $\rho(E, u, \Omega) \leq \gamma u^{q}, u>0$, where $1<q \leq 2$. If for a given $\epsilon>0$, there is an element $\varphi^{\epsilon} \in \mathcal{D}$, such that

$$
E\left(\varphi^{\epsilon}\right) \leq \inf _{x \in \Omega} E(x)+\epsilon, \quad \varphi^{\epsilon} / A(\epsilon) \in A_{1}(\mathcal{D})
$$

for some constant $A(\epsilon) \geq 1$, then, the output $x_{m}^{\mathrm{w}}$ of the WCGA satisfies the inequality

$$
E\left(x_{m}^{\mathrm{w}}\right)-\inf _{x \in \Omega} E(x) \leq \max \left\{2 \epsilon, C_{1} A(\epsilon)^{q}\left(C_{2}+\Sigma_{k=1}^{m} t_{k}^{q /(q-1)}\right)^{1-q}\right\},
$$

with constants $C_{1}=C_{1}(q, \gamma)$ and $C_{2}=C_{2}(E, q, \gamma)$.

\section{Main Results}

In this section, we present our main results and the auxiliary lemmas, needed for their proof. First, note that the set $\Omega:=\{x \in H: E(x) \leq E(0)\}$ is convex since it is the level set of a convex function. Also, all outputs $\left\{x_{k}\right\}_{k=1}^{\infty}$ generated by the $\mathrm{OMP}(\mathrm{co})$ (or the WCGA $(\mathrm{co}))$ are in $\Omega$, since the sequence $\left\{E\left(x_{k}\right)\right\}_{k=1}^{\infty}$ is decreasing and $E\left(x_{1}\right) \leq E(0)$.

\subsection{Auxiliary lemmas.}

Here, we begin with some lemmas that we use to derive our main results. The next lemma is well-known.

Lemma 4.1. Let $F$ be a Frechet differentiable function. Let $V_{k}:=\operatorname{span}\left\{\varphi_{j_{1}}, \ldots, \varphi_{j_{k}}\right\}$ and $x_{k}:=\operatorname{argmin}\left\{F(x): x \in V_{k}\right\}$. Then, we have that $\left\langle F^{\prime}\left(x_{k}\right), \varphi\right\rangle=0$ for every $\varphi \in V_{k}$. 
Our next lemma can be viewed as a generalization of Lemma 2.16 from [9].

Lemma 4.2. Let $\ell>0, r>0, B>0,\left\{a_{m}\right\}_{m=1}^{\infty}$ and $\left\{r_{m}\right\}_{m=2}^{\infty}$ be sequences of non-negative numbers satisfying the inequalities

$$
a_{1} \leq B, \quad a_{m+1} \leq a_{m}\left(1-\frac{r_{m+1}}{r} a_{m}^{\ell}\right), \quad m=1,2, \ldots
$$

Then, we have

$$
a_{m} \leq \max \left\{1, \ell^{-1 / \ell}\right\} r^{1 / \ell}\left(r B^{-\ell}+\sum_{k=2}^{m} r_{k}\right)^{-1 / \ell}, \quad m=2,3, \ldots
$$

Proof. Let us first notice that from the recursive relation and the fact that all $a_{m}$ 's are non-negative, we have

$$
0 \leq 1-\frac{r_{m+1}}{r} a_{m}^{\ell} \leq 1, \quad m=1,2, \ldots
$$

We will show that for $m=2,3, \ldots$.

$$
a_{m}^{\ell} \leq \begin{cases}\frac{r}{\left(r B^{-\ell}+\sum_{k=2}^{m} r_{k}\right)}, & \text { if } \ell \geq 1, \\ \frac{r}{\left(r B^{-\ell}+\ell \sum_{k=2}^{m} r_{k}\right)}, & \text { if } 0<\ell \leq 1,\end{cases}
$$

from which the inequality (4.13) easily follows.

We prove (4.15) by induction.

Case 1: $\ell \geq 1$.

If $a_{2}=0$, then all $a_{m}=0, m=3,4, \ldots$, and the lemma is true. Let us assume that $a_{2}>0$, and therefore $a_{1}>0$. It follows from the recursive relation and (4.14) that for $\ell \geq 1$

$$
a_{2}^{-\ell} \geq a_{1}^{-\ell}\left(1-\frac{r_{2}}{r} a_{1}^{\ell}\right)^{-\ell} \geq a_{1}^{-\ell}\left(1-\frac{r_{2}}{r} a_{1}^{\ell}\right)^{-1} \geq a_{1}^{-\ell}\left(1+\frac{r_{2}}{r} a_{1}^{\ell}\right)=a_{1}^{-\ell}+\frac{r_{2}}{r} \geq B^{-\ell}+\frac{r_{2}}{r} .
$$

This gives (4.15) for $m=2$.

We now assume that (4.15) is true for $m$ and prove it's validity for $m+1$. As in the case $m=2$, we may assume that $a_{m+1}>0$. Because of the recursive relation, this also means that $a_{m}>0$ and using (4.14), we derive

$$
a_{m+1}^{-\ell} \geq a_{m}^{-\ell}\left(1-\frac{r_{m+1}}{r} a_{m}^{\ell}\right)^{-\ell} \geq a_{m}^{-\ell}\left(1+\frac{r_{m+1}}{r} a_{m}^{\ell}\right)=a_{m}^{-\ell}+\frac{r_{m+1}}{r} .
$$

Now, from the induction hypothesis we have that

$$
a_{m}^{-\ell} \geq \frac{r B^{-\ell}+\sum_{k=2}^{m} r_{k}}{r}
$$

which combined with (4.16) proves the lemma in the case $\ell \geq 1$.

Case 2: $0<\ell<1$. 
Again, we need only consider the case when $a_{2}>0$. We will use the fact that for $0<\ell<1$, the function $(1-t)^{\ell}$ is concave. Therefore, we have

$$
(1-t)^{\ell} \leq 1-\ell t, \quad 0 \leq t \leq 1 .
$$

We apply this inequality with $t=\frac{r_{2}}{r} a_{1}^{\ell} \in[0,1]$ and obtain

$$
\begin{aligned}
a_{2}^{-\ell} & \geq a_{1}^{-\ell}\left(1-\frac{r_{2}}{r} a_{1}^{\ell}\right)^{-\ell} \geq a_{1}^{-\ell}\left(1-\ell \frac{r_{2}}{r} a_{1}^{\ell}\right)^{-1} \geq a_{1}^{-\ell}\left(1+\ell \frac{r_{2}}{r} a_{1}^{\ell}\right) \\
& =a_{1}^{-\ell}+\ell \frac{r_{2}}{r} \geq B^{-\ell}+\ell \frac{r_{2}}{r},
\end{aligned}
$$

which gives (4.15) for $m=2$. Next, we assume that (4.15) is true for $m$ and prove it for $m+1$. We can assume $a_{m+1}>0$ and therefore $a_{m}>0$. From the recursive relation and (4.17) with $t=\frac{r_{m+1}}{r} a_{m}^{\ell} \in[0,1]$, we have

$$
\begin{aligned}
a_{m+1}^{-\ell} & \geq a_{m}^{-\ell}\left(1-\frac{r_{m+1}}{r} a_{m}^{\ell}\right)^{-\ell} \geq a_{m}^{-\ell}\left(1-\ell \frac{r_{m+1}}{r} a_{m}^{\ell}\right)^{-1} \\
& \geq a_{m}^{-\ell}\left(1+\ell \frac{r_{m+1}}{r} a_{m}^{\ell}\right)=a_{m}^{-\ell}+\ell \frac{r_{m+1}}{r} .
\end{aligned}
$$

This inequality, combined with the induction hypothesis gives that

$$
a_{m+1}^{-\ell} \geq \frac{r B^{-\ell}+\ell \Sigma_{k=2}^{m+1} r_{k}}{r}
$$

and the proof is complete.

\subsection{Convergence rates for $\mathrm{OMP}(\mathbf{c o})$.}

In this section, we analyze the performance of the $\mathrm{OMP}(\mathrm{co})$ algorithm when applied to the minimization problem (1.1) with $D=H$. We assume that the dictionary $\mathcal{D}$ is an orthonormal system $\left\{\varphi_{i}\right\}_{i=1}^{\infty}$ and $E$ takes on its global minimum $\bar{x}$. This means that this global minimum is assumed over $\Omega:=\{x: E(x) \leq E(0)\}$. Let us denote by $e_{k}$ the error of the algorithm at Step k, namely,

$$
e_{k}:=E\left(x_{k}\right)-E(\bar{x}) \text {. }
$$

The next lemma provides a recursive relation for the sequence $\left\{e_{k}\right\}_{k=1}^{\infty}$.

Lemma 4.3. Let the objective function E satisfy Conditions $\mathbf{0}, \mathbf{1}$, and $\mathbf{2}$, and $\mu$ be a constant such that $\mu>\max \left\{1, M_{0} \alpha^{-1} M^{1-q}\right\}$. Let problem (1.1) have a solution $\bar{x}=\sum_{i} c_{i}(\bar{x}) \varphi_{i} \in \Omega$ with support $\bar{S}:=\left\{i: c_{i}(\bar{x}) \neq 0\right\}<\infty$, where $\left\{\varphi_{i}\right\}$ is an orthonormal basis. Then, the error of the $O M P(c o)$ applied to $E$ and $\left\{\varphi_{i}\right\}$ satisfies the following recursive inequalities:

$$
e_{1} \leq E(0)-E(\bar{x})
$$

and

$$
e_{k} \leq e_{k-1}-\frac{(\mu-1) \mu^{-q /(q-1)}}{r} e_{k-1}^{\frac{(p-1) q}{(q-1) p}}, \quad k \geq 2
$$


where the constant $r$ is

$$
r=|\bar{S}|^{\frac{q}{2(q-1)}} \alpha^{\frac{1}{q-1}}\left(p \beta_{0}^{1 / p}(p-1)^{(1-p) / p}\right)^{-q /(q-1)} .
$$

Proof. Clearly, we have $e_{1}=E\left(x_{1}\right)-E(\bar{x}) \leq E(0)-E(\bar{x})$ since

$$
x_{1}:=\operatorname{argmin}_{x \in \operatorname{span}\left\{\varphi_{j_{1}}\right\}} E(x) .
$$

Next, we consider Step $\mathrm{k}, k=2,3, \ldots$ of the algorithm. Observe that if at Step $(\mathrm{k}-1)$ we have that $\bar{S} \subseteq\left\{j_{1}, \ldots, j_{k-1}\right\}$, then $x_{k-1}=\bar{x}, E^{\prime}\left(x_{k-1}\right)=0$ and the $\operatorname{OMP}(\mathrm{co})$ would have stopped with output $x_{k-1}=\bar{x}$. If the algorithm has not stopped, then it generates the next output $x_{k}$ and $\varphi_{j_{k}}$. Since $x_{k}$ is the point of minimum of $E$ over $\operatorname{span}\left\{\varphi_{j_{1}}, \varphi_{j_{2}}, \ldots, \varphi_{j_{k}}\right\}$, we have for any $|t| \leq M$,

$$
E\left(x_{k}\right) \leq E\left(x_{k-1}+t \varphi_{j_{k}}\right) \leq E\left(x_{k-1}\right)+t\left\langle E^{\prime}\left(x_{k-1}\right), \varphi_{j_{k}}\right\rangle+\alpha|t|^{q},
$$

where the last inequality invoked Condition 1. We take

$$
t=-(\alpha \mu)^{-\frac{1}{q-1}} \operatorname{sign}\left(\left\langle E^{\prime}\left(x_{k-1}\right), \varphi_{j_{k}}\right\rangle\right)\left|\left\langle E^{\prime}\left(x_{k-1}\right), \varphi_{j_{k}}\right\rangle\right|^{\frac{1}{q-1}} .
$$

Because of the definition of $\mu$ in the statement of the theorem, we have $|t| \leq M$. Therefore, we have

$$
E\left(x_{k}\right) \leq E\left(x_{k-1}\right)-\frac{\mu-1}{\mu}(\alpha \mu)^{-\frac{1}{q-1}}\left|\left\langle E^{\prime}\left(x_{k-1}\right), \varphi_{j_{k}}\right\rangle\right|^{q /(q-1)} .
$$

Now, we will find a lower bound for $\left|\left\langle E^{\prime}\left(x_{k-1}\right), \varphi_{j_{k}}\right\rangle\right|$. First, note that from Condition 2 and Claim 1 applied to $x^{\prime}=\bar{x}$ and $x=x_{k-1}$ (both are in $\Omega$ ), we obtain

$$
\left\langle E^{\prime}\left(x_{k-1}\right), x_{k-1}-\bar{x}\right\rangle \geq E\left(x_{k-1}\right)-E(\bar{x})+\beta_{0}\left\|\bar{x}-x_{k-1}\right\|^{p} .
$$

Let us recall the weighted arithmetic mean -geometric mean inequality

$$
\frac{p_{1}}{p_{1}+p_{2}} a+\frac{p_{2}}{p_{1}+p_{2}} b \geq a^{\frac{p_{1}}{p_{1}+p_{2}}} b^{\frac{p_{2}}{p_{1}+p_{2}}}, \quad \text { where } \quad a, b \geq 0, \quad p_{1}, p_{2}>0
$$

and apply it for $p_{1}=p-1, p_{2}=1, a=\frac{E\left(x_{k-1}\right)-E(\bar{x})}{p-1}, b=\beta_{0}\left\|\bar{x}-x_{k-1}\right\|^{p}$. We have $E\left(x_{k-1}\right)-E(\bar{x})+\beta_{0}\left\|\bar{x}-x_{k-1}\right\|^{p}=p\left(\frac{(p-1)}{p} \frac{E\left(x_{k-1}\right)-E(\bar{x})}{p-1}+\frac{1}{p} \beta_{0}\left\|\bar{x}-x_{k-1}\right\|^{p}\right)$, and therefore

$$
E\left(x_{k-1}\right)-E(\bar{x})+\beta_{0}\left\|\bar{x}-x_{k-1}\right\|^{p} \geq C\left\|\bar{x}-x_{k-1}\right\|\left(E\left(x_{k-1}\right)-E(\bar{x})\right)^{(p-1) / p},
$$

with $C=p \beta_{0}^{1 / p}(p-1)^{(1-p) / p}$. We combine this inequality with (4.22) to obtain

$$
\left\langle E^{\prime}\left(x_{k-1}\right), x_{k-1}-\bar{x}\right\rangle \geq C\left\|\bar{x}-x_{k-1}\right\|\left(E\left(x_{k-1}\right)-E(\bar{x})\right)^{(p-1) / p} .
$$

From the definition of $x_{k-1}$ and Lemma 4.1, it follows that

$$
\left\langle E^{\prime}\left(x_{k-1}\right), \varphi_{i}\right\rangle=0, \quad i=j_{1}, \ldots, j_{k-1} \text {. }
$$


GREEDY STRATEGIES FOR CONVEX OPTIMIZATION

Therefore, if we write

$$
x_{k-1}-\bar{x}=\sum_{i} c_{i}\left(x_{k-1}-\bar{x}\right) \varphi_{i}
$$

since the support of $x_{k-1}$ is $\left\{j_{1}, \ldots, j_{k-1}\right\}$, we obtain

$$
\begin{aligned}
\left\langle E^{\prime}\left(x_{k-1}\right), x_{k-1}-\bar{x}\right\rangle & =\sum_{i \in \bar{S} \backslash\left\{j_{1}, \ldots, j_{k-1}\right\}} c_{i}\left(x_{k-1}-\bar{x}\right)\left\langle E^{\prime}\left(x_{k-1}\right), \varphi_{i}\right\rangle, \\
& \leq \sum_{i \in \bar{S} \backslash\left\{j_{1}, \ldots, j_{k-1}\right\}}\left|c_{i}\left(x_{k-1}-\bar{x}\right)\right|\left|\left\langle E^{\prime}\left(x_{k-1}\right), \varphi_{j_{k}}\right\rangle\right| \\
& \leq\left|\left\langle E^{\prime}\left(x_{k-1}\right), \varphi_{j_{k}}\right\rangle\right||\bar{S}|^{1 / 2}\left\|x_{k-1}-\bar{x}\right\| .
\end{aligned}
$$

We combine this inequality with (4.23) and derive that

$$
\left|\left\langle E^{\prime}\left(x_{k-1}\right), \varphi_{j_{k}}\right\rangle\right|\left\|\bar{x}-x_{k-1}\right\||\bar{S}|^{1 / 2} \geq C\left\|\bar{x}-x_{k-1}\right\|\left(E\left(x_{k-1}\right)-E(\bar{x})\right)^{(p-1) / p} .
$$

Therefore we have the desired lower bound

$$
\left|\left\langle E^{\prime}\left(x_{k-1}\right), \varphi_{j_{k}}\right\rangle\right| \geq C|\bar{S}|^{-1 / 2}\left(E\left(x_{k-1}\right)-E(\bar{x})\right)^{(p-1) / p} .
$$

The latter result and (4.21) gives the estimate

$$
E\left(x_{k}\right) \leq E\left(x_{k-1}\right)-\frac{(\mu-1) C^{q /(q-1)}}{\mu^{q /(q-1)} \alpha^{1 /(q-1)}|\bar{S}|^{\frac{q}{2(q-1)}}}\left(E\left(x_{k-1}\right)-E(\bar{x})\right)^{\frac{(p-1) q}{(q-1) p}} .
$$

Subtracting $E(\bar{x})$ from both sides of this inequality results in (4.19) and the proof is completed.

We next remark that we can take a specific value for $\mu$ in the last lemma.

Remark 4.4. Let the objective function E satisfy Conditions 0, 1, and 2. Let problem (1.1) have a solution $\bar{x}=\sum_{i} c_{i}(\bar{x}) \varphi_{i} \in \Omega$ with support $\bar{S}:=\left\{i: c_{i}(\bar{x}) \neq\right.$ $0\}<\infty$, where $\left\{\varphi_{i}\right\}$ is an orthonormal basis. Then, the error of the $\mathrm{OMP}($ co) applied to $E$ and $\left\{\varphi_{i}\right\}$ satisfies the following recursive inequalities:

$$
e_{1} \leq E(0)-E(\bar{x})
$$

and

$$
e_{k} \leq e_{k-1}-\frac{C_{3}}{r} e_{k-1}^{\frac{(p-1) q}{(q-1) p}}=e_{k-1}\left[1-\frac{C_{3}}{r} e_{k-1}^{\frac{p-q}{(q-1) p}}\right], \quad k \geq 2,
$$

where $r$ is the constant from Lemma 4.3 and $C_{3}=C_{3}\left(M_{0}, M, \alpha, q\right)$ is

$$
C_{3}=\left\{\begin{array}{cc}
(q-1) q^{-q /(q-1)}, & \text { if } M_{0} M^{1-q} \alpha^{-1}<q, \\
\left(M_{0} M^{1-q} \alpha^{-1}-1\right) M_{0}^{-q /(q-1)} M^{-q} \alpha^{q /(q-1)}, & \text { if } \quad M_{0} M^{1-q} \alpha^{-1} \geq q .
\end{array}\right.
$$


Proof. The estimate follows from Lemma 4.3 and the fact that the function

$$
g(\mu)=(\mu-1) \mu^{-q /(q-1)}
$$

is increasing on $(1, q)$ and decreasing on $(q, \infty)$ with global maximum at $\mu=q$.

The next theorem is our main result about the $\mathrm{OMP}(\mathrm{co})$ algorithm.

Theorem 4.5. Let the objective function $E$ satisfy Conditions $\mathbf{0}, \mathbf{1}$, and $\mathbf{2}$. Let problem (1.1) with $D=\Omega:=\{x: E(x) \leq E(0)\}$ have a solution $\bar{x}=\sum_{i} c_{i}(\bar{x}) \varphi_{i} \in \Omega$ with support $\bar{S}:=\left\{i: c_{i}(\bar{x}) \neq 0\right\}<\infty$, where $\left\{\varphi_{i}\right\}$ is an orthonormal basis for $H$. Then, at Step $k$, the $O M P(c o)$ applied to $E$ and $\left\{\varphi_{i}\right\}$ outputs $x_{k}$, where either $x_{k}=\bar{x}$, in which case $e_{k}=0$, or:

(i) When $p \neq q$, for $k=2,3, \ldots$,

$$
\begin{gathered}
e_{k} \leq C|\bar{S}|^{\frac{p q}{2(p-q)}} k^{-\frac{p(q-1)}{p-q}} \\
\left\|x_{k}-\bar{x}\right\| \leq C^{\prime}|\bar{S}|^{\frac{q}{2(p-q)}} k^{-\frac{q-1}{p-q}}
\end{gathered}
$$

where $C$ and $C^{\prime}$ depend only on $p, q, \alpha, \beta, E$.

(ii) When $p=q=2$, we have the exponential decay

$$
\begin{gathered}
e_{k} \leq C_{2} \gamma^{k-1}, \\
\left\|x_{k}-\bar{x}\right\| \leq C_{2}^{\frac{1}{2}} \beta_{0}^{-\frac{1}{2}} \gamma^{(k-1) / 2}, \quad k=2,3, \ldots,
\end{gathered}
$$

where $\gamma:=1-\frac{\tilde{C}_{3}}{|S|}$ is in $(0,1), C_{2}=E(0)-E(\bar{x})$, and $\tilde{C}_{3}$ is a constant that depends on $\alpha, \beta$, and $E$.

Proof. In the case $p \neq q$, we define the sequence of non-negative numbers

$$
r_{k}=C_{3}, \quad a_{k}=E\left(x_{k}\right)-E(\bar{x}), \quad k=1,2, \ldots,
$$

and the numbers

$$
\begin{gathered}
r=|\bar{S}|^{\frac{q}{2(q-1)}} \alpha^{\frac{1}{q-1}}\left(p \beta^{1 / p}(p-1)^{(1-p) / p}\right)^{-q /(q-1)}>0, \\
\ell=\frac{p-q}{p(q-1)}>0, \quad B=E(0)-E(\bar{x})>0 .
\end{gathered}
$$

It follows from Remark 4.4 that the above defined sequences satisfy the conditions of Lemma 4.2, and therefore we have

$$
e_{k}=E\left(x_{k}\right)-E(\bar{x}) \leq C_{0}\left(\frac{|\bar{S}|^{\frac{q}{2(q-1)}}}{C_{1}|\bar{S}|^{\frac{q}{2(q-1)}}+C_{3}(k-1)}\right)^{\frac{p(q-1)}{p-q}}
$$

where

$$
C_{0}=C_{0}(p, q, \alpha, \beta)=\alpha^{\frac{p}{p-q}}\left(p \beta^{1 / p}(p-1)^{(1-p) / p}\right)^{-\frac{p q}{p-q}} \cdot \max \left\{1,\left(\frac{p(q-1)}{p-q}\right)^{\frac{p(q-1)}{p-q}}\right\}
$$


and

$$
C_{1}=C_{1}(p, q, \alpha, \beta, E)=\alpha^{\frac{1}{q-1}}\left(p \beta^{1 / p}(p-1)^{(1-p) / p}\right)^{-q /(q-1)}(E(0)-E(\bar{x}))^{\frac{q-p}{p(q-1)}} .
$$

One easily derives the estimate for $e_{k}$ in (i) from (4.27). The estimate for $\left\|x_{k}-\bar{x}\right\|$ in (i) now follows from Condition 2 with $x^{\prime}=x_{k}, x=\bar{x}$ and Lemma 3.1 .

In the case $p=q=2$, as before $E\left(x_{1}\right)-E(\bar{x}) \leq E(0)-E(\bar{x})$, and Lemma 4.3 and Remark 4.4 give that

$$
E\left(x_{k}\right)-E(\bar{x}) \leq\left(1-\frac{\tilde{C}_{3}}{|\bar{S}|}\right)\left(E\left(x_{k-1}\right)-E(\bar{x})\right), \quad k=2,3, \ldots,
$$

where

$$
\tilde{C}_{3}=\left\{\begin{array}{cc}
\frac{\beta_{0}}{\alpha}, & \text { if } M_{0} M^{-1} \alpha^{-1}<2, \\
4 \beta_{0}\left(M_{0} M^{-1} \alpha^{-1}-1\right) M_{0}^{-2} M^{-2} \alpha, & \text { if } \quad M_{0} M^{-1} \alpha^{-1} \geq 2 .
\end{array}\right.
$$

It follows that

$$
E\left(x_{k}\right)-E(\bar{x}) \leq(E(0)-E(\bar{x}))\left(1-\frac{\tilde{C}_{3}}{|\bar{S}|}\right)^{k-1}, \quad k=2,3, \ldots
$$

As in the previous case, we use Condition 2 with $x^{\prime}=x_{k}, x=\bar{x}$ and Lemma 3.1 to derive the estimate for $\left\|x_{k}-\bar{x}\right\|$.

4.3. Main results for WCGA(co). The convergence analysis of the WCGA(co) is almost the same as that for the $\mathrm{OMP}(\mathrm{co})$. We omit the details here and just state the error estimates for

$$
e_{k}^{\mathrm{w}}:=E\left(x_{k}^{\mathrm{w}}\right)-E(\bar{x})
$$

pointing out the main differences in the proof.

Theorem 4.6. Let the objective function $E$ satisfy Conditions $\mathbf{0}, \mathbf{1}$, and 2. Let problem (1.1) with $D=\Omega=\{x: E(x) \leq E(0)\}$ have a solution $\bar{x}=\sum_{i} c_{i}(\bar{x}) \varphi_{i} \in \Omega$ with support $\bar{S}:=\left\{i: c_{i}(\bar{x}) \neq 0\right\}<\infty$, where $\left\{\varphi_{i}\right\}$ is an orthonormal basis. Then, at Step $k$, the WCGA applied to $E$ and $\left\{\varphi_{i}\right\}$ outputs $x_{k}^{\mathrm{w}}$, where either $x_{k}^{\mathrm{w}}=\bar{x}$, in which case $e_{k}^{\mathrm{w}}=0$, or:

(i) When $p \neq q$, for each $k=2,3, \ldots$, we have

$$
e_{k}^{\mathrm{w}} \leq \tilde{C}|\bar{S}|^{\frac{p q}{2(p-q)}}\left(\sum_{j=2}^{k} t_{j}^{\frac{q}{q-1}}\right)^{\frac{p(q-1)}{p-q}}
$$




$$
\left\|x_{k}^{\mathrm{W}}-\bar{x}\right\| \leq \tilde{C}^{\prime}|\bar{S}|^{\frac{q}{2(p-q)}}\left(\sum_{j=2}^{k} t_{j}^{\frac{q}{q-1}}\right)^{\frac{(q-1)}{p-q}}
$$

where $\tilde{C}$ and $\tilde{C}^{\prime}$ depend only on $p, q, \alpha, \beta, E$.

(ii) When $p=q=2$, we have

$$
\begin{gathered}
e_{k}^{\mathrm{w}} \leq C_{2} \prod_{j=2}^{k}\left(1-\frac{\tilde{C}_{3}}{|\bar{S}|} t_{j}^{2}\right) \\
\left\|x_{k}^{\mathrm{w}}-\bar{x}\right\| \leq C_{2}^{\frac{1}{2}} \beta^{-\frac{1}{2}} \prod_{j=2}^{k}\left(1-\frac{\tilde{C}_{3}}{|\bar{S}|} t_{j}^{2}\right)^{1 / 2},
\end{gathered}
$$

with $C_{2}=E(0)-E(\bar{x})$ and $\tilde{C}_{3}$ depends on $\alpha, \beta$, and $E$.

Proof. The proof follows the lines of that of Theorem 4.5 and the corresponding lemmas. The difference is that instead of estimate (4.24), we have

$$
\begin{aligned}
\left\langle E^{\prime}\left(x_{k-1}^{\mathrm{w}}\right), x_{k-1}^{\mathrm{w}}-\bar{x}\right\rangle & =\Sigma_{i \in \bar{S} \backslash j_{1}, \ldots, j_{k-1}} c_{i}\left(x_{k-1}^{\mathrm{w}}-\bar{x}\right)\left\langle E^{\prime}\left(x_{k-1}^{\mathrm{w}}\right), \varphi_{i}\right\rangle \\
& \leq \Sigma_{i \in \bar{S} \backslash j_{1}, \ldots, j_{k-1}}\left|c_{i}\left(x_{k-1}^{\mathrm{w}}-\bar{x}\right)\right|\left|\left\langle E^{\prime}\left(x_{k-1}^{\mathrm{w}}\right), \varphi_{i}\right\rangle\right| \\
& \leq t_{k}^{-1}\left|\left\langle E^{\prime}\left(x_{k-1}^{\mathrm{w}}\right), \varphi_{j_{k}}\right\rangle\right| \Sigma_{i \in \bar{S}}\left|c_{i}\left(x_{k-1}^{\mathrm{w}}-\bar{x}\right)\right| \\
& \leq t_{k}^{-1}\left|\left\langle E^{\prime}\left(x_{k-1}^{\mathrm{w}}\right), \varphi_{j_{k}}\right\rangle\right|\left\|\bar{x}-x_{k-1}^{\mathrm{w}}\right\||\bar{S}|^{1 / 2},
\end{aligned}
$$

and that we use Lemma 4.2 with $r_{k}=C_{3} t^{\frac{q}{q-1}}, k=2,3, \ldots$

\section{REFERENCES}

[1] J. Borwein, A. Guiro, P. Hajek, and J. Vanderwerff, Uniformly convex functions on Banach Spaces, Proc. Amer. Math. Soc., 137, 1081-1091, 2009.

[2] S. Boyd, L. Vandenberghe, Convex optimization, Cambridge University Press, 2009.

[3] E. Candes and T. Tao, Decoding by linear programming, IEEE Trans. Inf. Theory, 51(12), 4203-4215, 2005.

[4] R. DeVore, V. Temlyakov, Some remarks on greedy algorithms, Advances in Computational Math., 5, 173-187, 1996.

[5] D. Donoho, Compressed Sensing, IEEE Trans. Inf. Theory, 52(4), 1289-1306, 2006.

[6] S. Mallat, Z. Zhang, Matching Pursuits with Time-Frequency Dictionaries, IEEE Transactions on Signal Processing, —bf 41(12), 3397-3415, 1993.

[7] V. Temlyakov, Greedy expansions in convex optimization, preprint, arXiv:1206.0393.

[8] V. Temlyakov, Greedy approximation in convex optimization, preprint, arXiv:1206.0392.

[9] V. Temlyakov, Greedy approximation, Cambridge monographs on Applied and Computational Mathematics, Cambridge University Press, 2011.

[10] C. Zalinescu, Convex Analysis in General Vector Spaces, World Scientific Publishing Co. Inc., River Edge, NJ, 2002. 
[11] T. Zhang, Sequential greedy approximation for certain convex optimization problems, IEEE Transactions on Information Theory, 49(3), 682-691, 2003.

[12] T. Zhang, Sparse recovery with Orthogonal Matching Pursuit under RIP, IEEE Trans. Info. Th, 57, 5215-6221, 2011.

Hao Nguyen

Department of Mathematics, Texas A\&M University, College Station, TX 77843, USA

htnguyen@math.tamu.edu

Guergana Petrova

Department of Mathematics, Texas A\&M University, College Station, TX 77843, USA

gpetrova@math.tamu.edu 